Philosophie ANTIQUE

\section{Philosophie antique}

Problèmes, Renaissances, Usages

19 | 2019

L'épicurisme antique

\title{
Entre peripatos et kepos
}

Le quintum genus aristotélicien chez Cicéron et la quarta natura épicurienne chez Lucrèce

\section{Giulia Scalas}

\section{(2) OpenEdition}

1 Journals

Édition électronique

URL : https://journals.openedition.org/philosant/3119

DOI : 10.4000/philosant.3119

ISSN : 2648-2789

Éditeur

Éditions Vrin

\section{Édition imprimée}

Date de publication : 31 octobre 2019

Pagination : 85-115

ISBN : 978-2-7574-2534-3

ISSN : 1634-4561

\section{Référence électronique}

Giulia Scalas, "Entre peripatos et kepos », Philosophie antique [En ligne], 19 | 2019, mis en ligne le 31 octobre 2020, consulté le 02 décembre 2022. URL : http://journals.openedition.org/philosant/3119 DOI : https://doi.org/10.4000/philosant.3119

\section{(c) $($ ) $\odot \odot$}

Creative Commons - Attribution - Pas d'Utilisation Commerciale - Pas de Modification 4.0 International - CC BY-NC-ND 4.0

https://creativecommons.org/licenses/by-nc-nd/4.0/ 


\section{ENTRE PERIPATOS ET KEPOS \\ Le quintum genus aristotélicien chez Cicéron et la quarta natura épicurienne chez Lucrèce}

Giulia Scalas

Université de Lille, Faculté des Humanités

RÉSUMÉ. L'objectif de cette étude est d'examiner l'hypothèse selon laquelle la quarta natura, théorisée par Épicure pour rendre compte des activités de l'âme et décrite par Lucrèce (DRN III, 237-244), résulte de l'appropriation par Épicure d'un argument aristotélicien afin de répondre aux critiques d'Aristote à l'égard de la théorie démocritéenne de l'âme. Pour ce faire, on analysera le témoignage de Cicéron (Tusc. I, 10, 22) sur le quintum genus attribué à Aristote et on rendra compte des débats sur ses sources possibles. En confrontant le témoignage cicéronien avec les vers dans lesquels Lucrèce expose la théorie de la quatrième nature, on montrera les fortes ressemblances entre leurs structures argumentatives. Sur la base de la possible datation de la théorie de la quintessence et de son attribution au Péripatos du $\mathrm{II}^{\mathrm{e}}$ siècle av. J-C, on proposera d'expliquer cette similarité par l'appropriation par Lucrèce de l'argument par soustraction qui caractérise la théorie de la quintessence, à partir de laquelle il aurait conçu la théorie de la quatrième nature. Afin de vérifier l'exactitude de cette interprétation, on examinera l'hypothèse selon laquelle l'appropriation de cet argument, et donc la conception de la quatrième nature, remontent à Épicure, la paternité de l'argument par soustraction (à partir duquel les péripatéticiens postérieurs pourraient avoir conçu la théorie de la quintessence) étant alors à attribuer à Aristote. Pour ce faire, on analysera un passage du De Caelo (269a30-269b2) et un passage de La Génération des animaux (736b29-737a1) où l'argument par soustraction sert à introduire d'une part l'éther et d'autre par le pneuma. Ensuite, en s'appuyant sur l'analyse d'un passage du De Anima (405a5-7), on montrera que l'appropriation de cet argument pourrait s'expliquer par la tentative, de la part d'Épicure, de répondre à une critique aristotélicienne concernant la théorie démocritéenne de l'âme.

Summary. The aim of this paper is to show that Epicurus' notion of quarta natura emerges as a result of his appropriation of an Aristotelian argument against Democritus' theory of the soul. In order to do so, the paper will be organised as follows. As a starting point, I will consider Cicero's account of the quintum genus (Tusc. I, 10 22) and offer

Philosophie antique, ${ }^{\circ} 19$ (2019), 85-115 
an evaluation of scholarly debates about his attribution of the theory to Aristotle. By comparing Cicero's testimony with Lucretius' one (DRN III, 237-244), I will show that the two accounts retain strong resemblances as to their argumentative structures. In order to account for this closeness, I will offer two interpretative options. (i) On the basis of a later dating of the quintessence theory and its attribution to the Peripatos of the $2^{\text {nd }}$ century BCE, I will attempt an explanation by relying on Lucretius' own appropriation of the quintessence theory through an argument 'by subtraction'. Alternatively, (ii) I will examine whether the theory of the fourth nature should be acknowledged to Epicurus already, who borrows the argument by subtraction from Aristotle directly. This option will be verified on the basis of two Aristotelian passages (De Caelo, 296a30-269b2; Generation of Animals, 736629-737al), where the argument by subtraction introduces, respectively, the ether and the pneuma. Finally, through an analysis of De Anima, 405a 5-7, I will show that option (ii) has interpretative advantages, as it explains Epicurus attempt to counter an Aristotelian critique against Democritus' theory of the soul. 


\section{Introduction}

«L'âme est un corps »: par ces mots, Épicure introduit sa doctrine psychologique aux vers 63-67 de la Lettre à Hérodote. À la lumière de son projet philosophique, cette phrase s'avère particulièrement significative : non seulement parce qu'il s'agit d'une déclaration forte et en opposition ouverte à toutes les philosophies précédentes qui avaient insisté sur l'hétérogénéité de l'âme par rapport au/aux corps, mais surtout parce qu'elle représente de la façon la plus efficace le lien qui existe entre connaissance et bonheur. La connaissance ne concerne pas des objets intelligibles et éloignés, difficilement saisissables, mais plutôt les corps, leurs mouvements et les phénomènes plus ou moins visibles qui les caractérisent. Dans un discours qui est à l'opposé de ceux de la plupart de ses prédécesseurs, l'âme est, selon Épicure, un objet d'étude privilégié, non pas parce qu'elle serait supérieure par rapport aux autres corps, mais parce que c'est seulement en prenant conscience du fait que l'âme est un corps (comme tous les autres objets qui interagissent dans cet univers) que l'individu se libère des fausses opinions les plus dangereuses qui l'empêchent de vivre de façon heureuse : le désir d'immortalité et la peur de la mort. À cette phrase introductive qui ouvre le paragraphe sur la nature matérielle de l'âme $(\$ 63)$ correspond de façon spéculaire le dernier paragraphe de la section psychologique $(\$ 67)$ qui porte sur l'impossibilité que l'âme soit incorporelle. Entre-temps, Épicure montre de façon synthétique mais efficace la relation d'interdépendance entre l'âme et le corps. Comme l'ont justement reconnu D. Sedley et A. Long ${ }^{1}$, l'objectif d'Épicure dans la Lettre est en fait de démontrer la corporéité de l'âme par le moyen de son inséparabilité du corps, dans une stratégie qui semble être la même, mais inversée et poursuivant l'objectif contraire, que celle qui avait conduit Platon à démontrer la séparabilité de l'âme vis-à-vis du corps en insistant sur son incorporéité.

Une partie importante de la théorie psychologique d'Épicure a été

1. Long \& Sedley 1987, p. 71. 
reconstituée ${ }^{2}$ grâce au poème de Lucrèce et aux témoignages de doxographes et de philosophes plus tardifs. Il s'agit notamment de la partie consacrée aux activités proprement psychiques qui est presque complètement absente dans la Lettre. En effet, ces témoignages se révèlent particulièrement importants en vue d'une compréhension plus approfondie de l'explication matérialiste d'activités comme la pensée et la sensation. Parmi ces témoignages, un passage, celui d'Aëtius, est très utile pour résumer la théorie épicurienne de la constitution élémentaire de l'âme :

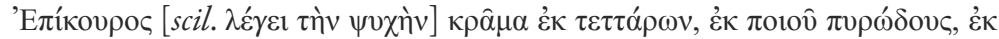

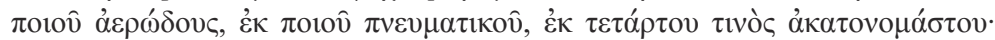

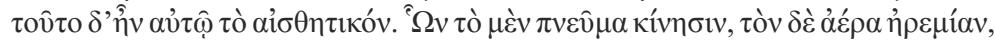

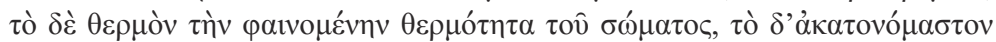

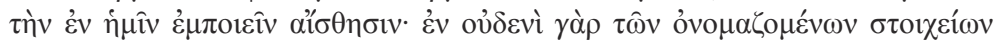

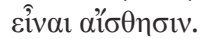

Épicure [dit que l'âme est] un mélange fait de quatre éléments, dont l'un ressemble au feu, l'autre à l'air, l'autre au souffle de vent, le quatrième n'ayant pas de nom. C'est à ce dernier qu'il attribue la responsabilité de la sensation. Le souffle de vent, dit-il, produit en nous le mouvement, l'air produit le repos, le chaud produit la chaleur manifeste du corps, et l'élément sans nom produit la sensation en nous, car celle-ci ne se trouve dans aucun des éléments qui ont un nom ${ }^{3}$.

Épicure aurait conçu une théorie prévoyant l'existence de quatre éléments qui, mélangés, constitueraient la nature de l'âme : la chaleur, le souffle, l'air et une quatrième nature sans nom. Ce dernier élément serait introduit afin d'expliquer comment l'âme peut être douée de sensation, étant donné que les trois autres éléments ne peuvent pas l'expliquer. Il s'agirait d'un élément, donc d'un corps, sans nom, capable de rendre compte des activités psychologiques principales. Cette théorie est confirmée par Lucrèce qui, comme on le verra, lui consacre de nombreux vers au livre III de son poème. Mais d'où cette théorie dérive-t-elle ? Elle n'est certainement pas démocritéenne ${ }^{4}$. Est-elle authentiquement épicurienne ? Dans son Aristotele perduto 5 , Bignone a été le premier à remarquer une certaine ressemblance entre les témoignages sur la quatrième nature d'Épicure et quatre passages des Tusculanae Disputationes de Cicéron ${ }^{6}$. À l'intérieur de son compte-rendu des psychologies de ses

2. Je ne m'occuperai pas ici des divergences entre les contenus des paragraphes 63-67 de la Lettre à Hérodote et le livre III du De rerum natura. Je renvoie donc à Kerferd 1971, p. 80-96 et à Verde 2015, p. 49-64.

3. Aëtius, 4.3.11 = Us. 315 (Trad. Brunschwig \& Pellegrin 2001).

4. Cf. Sassi 1978, p. 161-213; Morel 1996, p. 129-176; Taylor 1999, p. 200-211.

5. Bignone $1973^{2}$, p. 224 sq. Selon Bignone, Cicéron transmettrait une doctrine psychologique antérieure à celle du De Anima, appartenant à la première phase de la pensée aristotélicienne.

6. Tusc. I, 10, $22 ; \mathrm{I}, 17,41 ; \mathrm{I}, 26,65 ; \mathrm{I}, 27,66$. 
prédécesseurs, Cicéron résume entre autres celle d'Aristote. Ce dernier aurait tenu pour une cinquième essence sans nom la matière qui constitue l'âme et qui lui permet d'accomplir des actions comme penser, imaginer, apprendre, etc. À la suite de Bignone, c'est surtout Boyancé qui s'est interrogé sur une possible relation entre les deux doctrines : « sans doute ce quatrième élément n'est pas sans rappeler la fameuse quintessence d'Aristote et sa conception a pu en subir l'influence $\gg^{7}$. Cependant, tout en reconnaissant une certaine proximité entre les rôles joués par la quintessence et la quatrième nature, le savant exclut toute forme possible d'influence, en affirmant que la deuxième

differe radicalement (de la première) en ce qu'elle est tout de même de nature apparentée à celle du chaud, de l'air et du vent et comme eux composée d'atomes. Ce qui importe pour juger une doctrine comme celle-là, ce n'en est pas tant la lettre, que l'orientation de la pensée qui la formule. Cette orientation est vers le matérialisme, non vers le spiritualisme et rien ne permet de croire que Lucrèce pense dans un sens différent de celui d'Épicure ${ }^{8}$.

Le savant arrête ainsi son enquête, sitôt le rapprochement découvert. Or, malgré la différence d'orientation évoquée par Boyancé, la proximité entre les deux théories ne peut pas être minimisée et la comparaison mérite d'être approfondie : quelle est donc la relation entre la théorie de la quintessence attribuée par Cicéron à Aristote et celle de la quatrième nature épicurienne exposée par Lucrèce?

Je montrerai d'abord la proximité entre la théorie aristotélicienne exposée par Cicéron et celle, épicurienne, qu'on lit chez Lucrèce. Elle pourrait s'expliquer par l'appropriation de la part de Lucrèce d'un argument non épicurien certes, mais assez connu à l'époque. J'interrogerai ensuite la structure argumentative commune aux théories de la quatrième nature et à celle de la quintessence. Peut-on l'attribuer à Épicure, et ne pourrait-elle pas s'expliquer comme une appropriation d'un argument aristotélicien utilisé pour répondre dialectiquement à la critique que fait Aristote de la théorie psychologique démocritéenne?

\section{Le quintum genus d'Aristote chez Cicéron et la quarta natura chez Lucrèce}

C'est d'abord le témoignage sur Aristote que donne Cicéron dans les Tusculanes que j'observerai :

Aristoteles, longe omnibus (Platonem semper excipio) praestans et ingenio et diligentia, cum quattuor nota illa genera principiorum esset complexus, e quibus omnia orerentur, quintam quandam naturam censet esse, e qua sit mens; cogitare enim et providere et discere et docere et invenire aliquid et tam multa [alia] meminisse, amare, odisse, cupere, timere, angi, laetari, haec et similia eorum in 
horum quattuor generum inesse nullo putat; quintum genus adhibet vacans

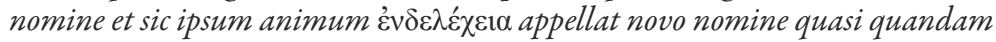
continuatam motionem et perennem.

Aristote, qui l'emporte de beaucoup sur tous les philosophes - j'excepte toujours Platon - et par l'esprit d'invention et par l'esprit de méthode, estime, après avoir étendu ses recherches à ces quatre catégories bien connues d'éléments d'où tous les êtres procéderaient, qu'il existe une cinquième essence spéciale d'où procède la pensée : imaginer, prévoir, apprendre, enseigner, inventer, se rappeler tant de choses, aimer et haïr, désirer et craindre, s'affliger et se réjouir et les fonctions analogues ne peuvent relever d'aucune de ces quatre catégories. Il a donc recours à une cinquième catégorie qui n'a pas de nom et est conduit, en ce qui concerne l'âme même, à la désigner par un terme nouveau, $\dot{\varepsilon} v \delta \varepsilon \lambda \varepsilon_{\varepsilon} \chi \varepsilon 1 \alpha$, ce qui revient à dire une espèce de mouvement ininterrompu et perpétuel ${ }^{9}$.

La théorie que nous transmet ici Cicéron n'a rien à voir avec la définition de l'âme comme forme d'un corps qui a la vie en puissance, qui est développée dans le De Anima ${ }^{10}$. Selon le philosophe romain, Aristote aurait considéré un cinquième élément comme étant la matière constitutive de l'âme, capable de rendre raison de toutes ses activités, comme penser, imaginer, aimer, etc. que les autres éléments n'auraient pu produire. La plupart des commentateurs

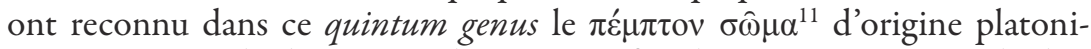
cienne, à savoir l'éther aristotélicien. En effet, il s'agit comme pour l'éther d'un élément qui s'ajoute aux quatre connus : air-feu-eau-terre. Dans un passage des Consolationes, cité dans les Tusculanes, Cicéron détaille un peu plus cette question en affirmant que

rien sur la terre ne saurait rendre compte de l'origine de l'âme, car dans l'âme il n'y a rien de mixte et de composé, et que l'âme ne renferme rien qui paraisse être né ou formé de la terre, rien non plus qui appartienne à l'élément liquide, à l'élément du souffle ou à l'élément du feu. Ces éléments en effet ne renferment rien qui comporte l'essence de la mémoire, de l'intelligence, de la réflexion ${ }^{12}$.

De plus, dans un autre passage, Cicéron affirme que cette nature est commune aux dieux et à l'âme ${ }^{13}$. Ces passages ont été au centre d'un débat important puisque Cicéron affirme avoir tiré ces informations du Peri Philo-

9. Tusc. I, 10, 22 (trad. Humbert 1931).

10. 412a 20-21.

11. Epin. 91c7. L'authenticité de ce dialogue est très douteuse. À ce propos, je renvoie à l'introduction de Harward 1928 ; Taràn 1975; Brisson 2005, p. 9-24.

12. Tusc. I, 27, 66: Animorum nulla in terris origo inveniri potest; nibil enim est in animis mixtum atque concretum aut quod ex terra natum atque fictum esse videatur, nibil ne aut umidum quidem aut flabile aut igneum. His enim in naturis nibil inest, quod vim memoriae, mentis, cogitationis habeat. (trad. Humbert 1931 légèrement modifiée pour mixtum atque concretum.)

13. Tusc. I, 26, 65. 
sophias d'Aristote. Ils se révèlent des éléments utiles pour la reconstitution de la philosophie de celui qu'il est convenu d'appeler le premier Aristote.

Sans entrer dans le détail de l'authenticité des témoignages et de la datation du Peri Philosophias, on peut résumer les deux principales positions interprétatives concernant la question de l'âme-éther. Pour les uns, la cinquième nature est l'éther ; pour les autres, la cinquième nature ne saurait être l'éther. Joseph Moreau, représentant de la première position, identifie le dieu du Peri

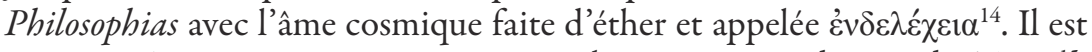
suivi par A.-J. Festugière qui voit cette théorie comme dérivée du Timée ${ }^{15}$. Plus précisément, sur la question psychologique, L. Alfosi ${ }^{16}$ et G. Soleri ${ }^{17}$ ont

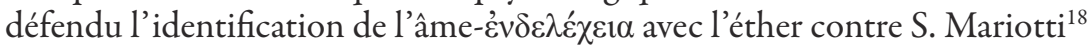
et A. Mansion ${ }^{19}$, qui refusent cette identification, puisqu'en dériverait une psychologie matérialiste incohérente avec l'hylémorphisme de l'Aristote de la maturité, mais aussi avec l'apparent spiritualisme de l'Aristote académicien. Karl Reinhardt ${ }^{20}$ est le premier à proposer une source intermédiaire entre Aristote et Cicéron, qui aurait transmis une version réélaborée des positions aristotéliciennes. Cette source serait le néo-académicien Antiochus d'Ascalon ${ }^{21}$. Plus ou moins sur la même ligne, D. Hahm² ${ }^{22}$ à travers une analyse détaillée des témoignages, exclut la possibilité que le cinquième élément ait été introduit par Aristote dans le Peri Philosophias, en réduisant l'importance de ces témoignages de Cicéron pour la reconstitution de cet ouvrage. Outre Antiochus, le savant propose, comme sources possibles de ces informations, Posidonius, mais aussi des recueils doxographiques largement employés à partir de l'époque hellénistique. Charles Brittain, au contraire, considère le contenu de ce témoignage comme le produit d'une réélaboration tardive : «Cicero's error is probably due to later Peripatetic elaborations of Aristotle's scattered remarks on the role of pneuma (fiery air) in human psycho$\log y$, and a subsequent conflation of pneuma with the fifth element $\gg{ }^{23}$. Au-delà des débats concernant le Peri Philosophias, l'identification entre le quintum genus et l'éther ${ }^{24}$ est généralement considérée à la lumière du contenu du $D e$

14. Moreau 1939, p. 106-127.

15. Festugière 1949, p. 219-259.

16. Alfonsi 1951, p. 71-78.

17. Soleri 1952, p. 67-68.

18. Mariotti 1940, p. 179-189.

19. Mansion 1953, p. 444-472.

20. Reinhardt 1953, col. 576-586.

21. Pour approfondir la figure d'Antiochus, je renvoie à Sedley 2012.

22. Hahm 1982, p. 60-74.

23. Brittain 2006, p. 97 n. 24.

24. L'éther, selon Aristote, est : éternel (Metaph. IX, 1050b 6-14), le premier élément (Cael. III, 1, 298b 6 ; Mete. I, 1, 338b 21 et 3, 339b 17), décrit comme corporel (Cael. I, 2, 269b4 ; 3, 269b30 ; 270b3-21 ; II, 7, 289a30 ; 12, 293a8 ; Mete. I, 3, 340a20 ; De An. II, 6, 418b9-13), décrit comme perceptible (Metaph. I, 9, 991 a10 ; XII 1, 1069a30-31), inaltérable, 
Caelo, même si cette expression n'appartient pas au lexique aristotélicien connu. En effet, Aristote appelle normalement l'éther $\tau$ ò $\pi \rho \hat{\omega} \tau o v \sigma \hat{\omega} \mu \alpha^{25}$ ou

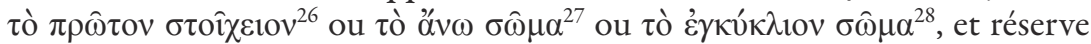
$\alpha i \theta n ́ \rho$ à l'éther selon les présocratiques ${ }^{29}$. Comme le remarque D. Hahm,

The only exception is Phys. IV 5.212b20-2 where Aristotle gives the stratification of the cosmos: "The earth is within the water; the water within the air ; the air within the ether; and the ether within the heaven; but the heaven is not in anything else." Here, when he does use the term 'ether', he does not use it of the fifth element, but rather of fire, a practice for which he chides Anaxagoras in Cael. I 3.270b24-5; III 3.302b4-5 $5^{30}$.

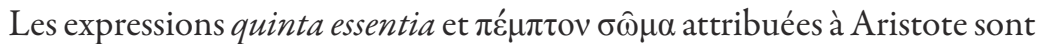
attestées dans la doxographie (Aëtius, II, 7, 5 p. 336, 12 D ; Ps.-Clément de Rome, Recognitiones, VIII, 15, 4, 2) et c'est probablement pour cette raison que Cicéron les emploie. Or, si l'on voulait soutenir l'identité du quintum genus et de l'éther, on rencontrerait sans aucun doute une difficulté : si ce cinquième élément est l'éther, pourquoi devrait-il être considéré par Aristote comme dépourvu de nom, comme le rapporte Cicéron ?

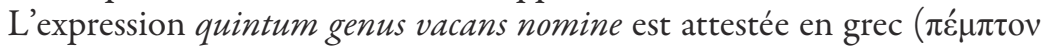
$\sigma \hat{\omega} \mu \alpha \dot{\alpha} \kappa \alpha \tau o v o ́ \mu \alpha \sigma \tau o v)$ chez le pseudo-Clément ${ }^{31}$ et attribuée à Aristote, mais il s'agit de la seule occurrence. Dans les ouvrages d'Aristote l'absence de nom de l'éther n'apparaît jamais. Cependant, le fait de ne pas nommer un objet, pourtant identifié et défini, n'est pas un comportement étranger à Aristote, bien au contraire. Il y a de nombreuses occurrences d'expressions comme oủ

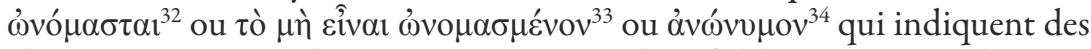
objets qui n'ont pas de nom mais qui sont identifiables et à propos desquels on peut tenir un discours. Les exemples les plus connus sont les comportements qui constituent un manque ou un excès au milieu desquels se trouve le comportement vertueux, dans le domaine éthique, et qui sont identifiables mais n'ont pas de nom ${ }^{35}$. Dans la Métaphysique, le cas du genre commun, à savoir le genre prochain, du cheval et de l'âne, n'a pas de nom ${ }^{36}$. Il est naturel,

comme n'augmentant ni ne diminuant (Cael. I, 3, 270b1-4 ; II, 6, 288a 34-b1), toujours en acte (Metaph. IX, 8, 1050b27-28).

25. Cael. $304 \mathrm{a} 24$.

26. Metaph. $988 \mathrm{a} 33$.

27. De An.418b13; Cael.289a17 ; MA 699b25.

28. Cael.286a12.

29. Par exemple Cael. $270 \mathrm{~b} 25$ et 294a25-26.

30. Hahm 1982, p. 62.

31. Clem. Recogn. VIII, 15, 4, 2.

32. EN 1127a7; Metaph. 1034al; Pol. 1253 b11.

33. De An. $74 \mathrm{a} 21$.

34. EE 1221a3; De An. 74a8.

35. EN $1127 \mathrm{a} 7$.

36. Metaph. $1034 \mathrm{a} 1$. 
cependant, de se demander pourquoi Aristote devrait considérer l'éther, élément déjà connu, identifié et nommé par les présocratiques, comme sans nom. E. Berti ${ }^{37}$ l'explique en faisant appel à la mention imprécise et sans nom du polyèdre qui constitue le cinquième élément dans le Timée ${ }^{38}$. Une autre explication possible est celle de D. Hahm, selon lequel

Aristotle's non-commital account of the traditional name 'ether' and his explicit criticism of Anaxagoras' use of this term (Cael. I3.270b16-25), combined with his own preference for descriptive paraphrases rather than a single name, is sufficient to account for the doxographical term ${ }^{39}$.

Dans ce cas-là, cette expression résumerait le choix d'Aristote de ne pas nommer cet élément comme les présocratiques pour se distinguer de leur conception et de s'en tenir généralement à l'expression « premier élément », une périphrase.

Revenons à Cicéron. L'idée selon laquelle Aristote concevrait l'âme comme constituée d'éther à l'instar des astres n'a évidemment pas de parallèle dans l'œuvre d'Aristote. Cependant une théorie de ce type est attribuée par Jamblique à certains aristotéliciens :

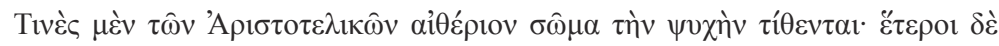

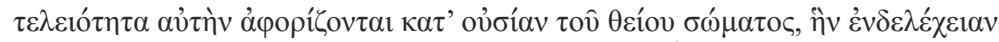

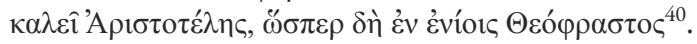

Certains aristotéliciens considèrent l'âme comme un corps fait d'éther. D'autres la définissent comme l'essence parfaite du corps divin, qu'Aristote appelle « mouvement perpétuel », comme Théophraste le fait aussi à certains endroits.

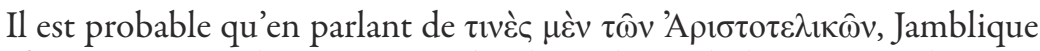
se réfere à ces aristotéliciens qui ont développé la psychologie aristotélicienne en suivant une orientation plus ou moins matérialiste. Une théorie de ce type est attribuée explicitement pour la première fois à Critolaos de Phasélis par Tertullien $^{41}$ et par Macrobe ${ }^{42}$, et aussi à Diodore de Tyr, par Aëtius ${ }^{43}$. La suite du témoignage est plus complexe. En effet, comme le remarquent à raison J. Finamore et J. M. Dillon dans leur commentaire du De Anima de Jamblique,

Here the presentation of the soul as "the essential perfection of the divine body" is linked to a term for it attributed by Theophrastus "Eे Ėviolc" (no doubt in his

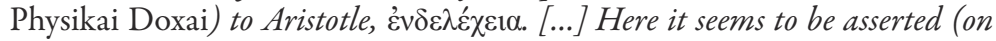

37. Berti 1962, p. 323.

38. Ti. 55c5.

39. Hahm 1982, p. 66 n. 23.

40. De An. 9, 2.

41. De An. $5,1$.

42. Comm. Somn. Scip. 1, 14, 20.

43. Aëtius I, 7, 21 p. 303 D. 
the authority of Theophrastus?) that the soul is taken to be somehow the essence and perfection of the heavenly realm, as if it were indeed its prote entelecheia, as well as being characterized by endelecheia, that is to say, perpetual self-motion. The linking of the terms teleiotes and endelecheia would lend substance to the hypothesis of a confusion between endelecheia and entelecheia, but it seems hardly possible that either Theophrastus or even Iamblichus himself (who knew the text of Aristotle's De Anima perfectly well) could have been guilty of such a confusion ${ }^{44}$.

Contrairement au témoignage de Cicéron, la théorie de l'âme-éther est ici distinguée de la théorie de l'âme-ร’ $v \delta \varepsilon \lambda \varepsilon ́ \chi \varepsilon 1 \alpha$. La première est attribuée à

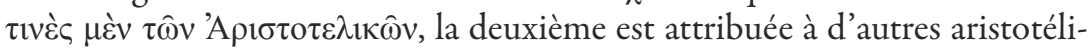
ciens, dont fait partie dans une certaine mesure Théophraste. Ces deux théories psychologiques n'appartiennent cependant pas directement à Aristote. Ce dernier en effet est mentionné par Jamblique seulement à propos du fait que, selon lui, l'essence parfaite du corps divin est appelée $\varepsilon \dot{v} \delta \varepsilon \lambda \varepsilon \varepsilon_{\chi} \varepsilon 1 \alpha$, à savoir mouvement perpétuel. Ce témoignage atteste donc l'existence de ces théories, en relation avec Aristote, mais sans possibilité de les lui attribuer avec certitude. Étant donné la rareté des témoignages, la paternité de la doctrine de l'âme-éther reste très discutée. Domine toutefois l'idée qu'Antiochus d'Ascalon serait à l'origine de cette idée de la cinquième nature. Dans son éclectisme, il aurait mélangé et réélaboré des théories platoniciennes et péripatéticiennes à la lumière de la doctrine stoïcienne ${ }^{45}$. Or, si on tient compte du témoignage de Jamblique, qui semble faire remonter une partie de la doctrine résumée par Cicéron à Théophraste, et si on identifie les aristotéliciens mentionnés avec Critolaos et Diodore de Tyr (qui ont vécu au II ${ }^{\mathrm{e}}$ siècle), l'hypothèse que la théorie du cinquième élément appartienne à Antiochus d'Ascalon semble peu vraisemblable. Si cette théorie ne semble pas non plus conçue par le premier Aristote, comme la plupart des spécialistes récents le pensent, il reste une solution : elle pourrait avoir été élaborée dans le Péripatos matérialiste post-aristotélicien, et peut-être transmise ensuite à Antiochus d'Ascalon, par l'intermédiaire duquel Cicéron pourrait l'avoir connue.

Venons-en maintenant à la théorie épicurienne de la quatrième nature. Le discours sur la constitution corporelle de l'âme, au livre III du De rerum natura de Lucrèce, est précédé par un examen des fonctions respectives de l'âme et du corps. Le début de l'exposé psychologique porte donc sur la distinction entre l'âme et le corps ${ }^{46}$. Cette distinction a pour objectif d'éviter toute interprétation éliminativiste du type de celle qui résulte des conceptions de l'âme comme harmonie du corps ${ }^{47}$. Lucrèce distingue donc l'âme

44. Finamore \& Dillon 2002, p. 96-97.

45. Cf. supra p. 91.

46. DRN III, v. 88-101.

47. Pour un regard plus approfondi sur la théorie de l'âme-harmonie, voir Della Valle 1905, p. 210-231; Gottschalk 1971, p. 179-198 ; Trabattoni 1988, p. 53-74; Caston 1997, 
et le corps en s'appuyant sur leurs fonctions différentes. Il souligne combien les deux natures, bien qu'unies et étroitement liées, peuvent se trouver dans deux états différents et contraires : ainsi quand le corps dort et que l'âme reste active dans le rêve ou encore quand le corps est malade, mais que l'âme est néanmoins heureuse. Établir une relation entre l'âme et le corps tout en maintenant l'indépendance de leurs activités, tel est l'objectif du raisonnement. Toujours sur la base de données dérivées de l'expérience, le poète ajoute ensuite que, même à l'intérieur de l'âme, il y a une distinction fonctionnelle et locale entre une partie rationnelle, l'animus, où se trouve la pensée et qui a son siège dans la poitrine, et une partie a-rationnelle, l'anima, principe de la sensation, qui est diffusée dans tout le $\operatorname{corps}^{48}$. Ces deux parties, tout en ayant deux sièges différents, sont cependant conjointes et forment une seule nature ${ }^{49}$. Mais de quelle nature s'agit-il ?

Lucrèce examine alors de plus près la composition matérielle de l'âme :

Tenvis enim quaedam moribundos deserit aura

mixta vapore, vapor porro trahit aera secum;

nec calor est quisquam, cui non sit mixtus et aer

rara quod eius enim constat natura, necessest

aeris inter eum primordia multa moveri.

Les mourants exhalent en effet un souffle ténu

Mêlé de chaleur, chaleur transportant de l'air à son tour,

Car il n'est point de chaleur qui ne soit mêlée d'air :

Sa nature poreuse implique nécessairement

De nombreux atomes d'air en mouvement ${ }^{50}$.

Toujours à partir de l'observation empirique, cette fois d'un homme mourant, Lucrèce identifie trois natures mêlées qui constituent l'âme : le souffle (aura), la chaleur (vapor) et l'air (aer). Les deux premières natures, le souffle et la chaleur, sont celles qui constituent de façon évidente le dernier souffle, l'air en revanche est identifié à partir d'un raisonnement : la consistance de la chaleur est trop rara (《 poreuse » dans la traduction) et doit donc « accueillir » de l'air.

Lucrèce reconnaît ensuite l'impossibilité que ces trois natures produisent ensemble la sensibilité :

iam triplex animi est igitur natura reperta;

nec tamen haec sat sunt ad sensum cuncta creandum,

p. 319-354.

48. À propos des activités et des différentes affections qui caractérisent l'animus et l'anima, voir Konstan 2007, p. 112-113.

49. DRN III, v. 137.

50. DRN III, v. 232-236 (trad. Kany-Turpin 1993). 
nil horum quoniam recipit mens posse creare

sensiferos motus, et mens quaecumque volutat ${ }^{51}$.

Quarta quoque his igitur quaedam natura necessest

adtribuatur; east omnino nominis expers;

qua neque mobilius quicquam neque tenvius extat

nec magis e parvis et levibus ex elementis ;

Voilà donc la triple nature de l'esprit découverte,

Mais ce n'est pas assez pour former la sensibilité

Car il est impensable qu'un de ces éléments

Puisse créer les mouvements perceptifs et mentaux ${ }^{52}$.

Il faut donc adjoindre une quatrième nature,

Mais cette nature n'a jamais reçu de nom.

Il n'est rien de plus mobile ni de plus ténu,

Rien dont les atomes soient plus petits ni plus lisses ${ }^{53}$.

Si ces trois natures étaient capables de donner naissance à la sensation, n'importe quel corps qui en serait composé pourrait percevoir et penser. Or, l'observation de la nature nous montre qu'il n'en va pas ainsi. Il faut donc supposer l'existence d'une quatrième nature capable de créer les sensiferos motus et la mens ${ }^{54}$. Dans ses vers sur le fonctionnement de l'esprit ${ }^{55}$, Lucrèce montre que la capacité de penser de l'homme est due à la finesse des parties qui composent son esprit. Le fonctionnement de la pensée et de la sensation sont expliquées par Épicure de la même façon : par la sensibilité au contact. Dans le premier cas, l'esprit est touché et mis en mouvement par les simulacra de la pensée. Dans le cas de la sensation ( $c f$. la démonstration du début du livre IV), l'objet perçu touche soit directement (pour le toucher et le goût) soit par la médiation des simulacra/masses (pour la vision, l'audition et l'odorat) l'âme éparpillée dans le corps ${ }^{56}$. Mais quel élément de l'âme est touché ? Et comment faut-il concevoir le fonctionnement de l'activité perceptive ? C'est sans aucun doute la quatrième nature qui est mise en mouvement ${ }^{57}$. Cette nature est caractérisée par une finesse extrême : les parties qui la composent

51. Le texte est problématique. Je suis la correction de Frerichs 1892.

52. Traduction Kany-Turpin 1993 modifiée.

53. DRN III, v. 237-244.

54. DRN III, v. 238.

55. DRN III, v. 175-185; IV, v. 722-815.

56. Les simulacres touchent l'âme après avoir traversé les pores. Sur la doctrine des pores, voir Leone 2002, p. 104-118.

57. Dans la suite du chant, la quatrième nature est définie comme « âme de toute l'âme » (anima est animae totius ipsa). En effet, elle est dite être cachée dans la profondeur du corps. Giussani 1896, p. 187-188, interprète très justement cette profondeur dans le sens d'une « intimité » matérielle. Étant donnée sa finesse, la quatrième nature occuperait les coins et 
sont les plus petites et les plus lisses présentes dans la nature. Cette finesse lui permet d'être mise en mouvement en premier dans l'action perceptive. Elle est la condition nécessaire pour qu'une sensation survienne.

Condition de cette « mise en mouvement », la quatrième nature ne peut pas accomplir sa fonction seule. Cela devient clair ensuite :

sensiferos motus quae didit prima per artus.

Prima cietur enim, parvis perfecta figuris,

inde calor motus et venti caeca potestas

accipit, inde aer, inde omnia mobilitantur :

concutitur sanguis, tum viscera persentiscunt

omnia, postremis datur ossibus atque medullis

sive voluptas est sive est contrarius ardor.

C'est la première à diffuser les mouvements sensitifs,

Car formée de petites figures elle s'émeut la première,

Puis la chaleur et la puissance aveugle du vent, puis l'air

Reçoivent ces mouvements, enfin tout se met en branle.

Le sang reçoit le choc et la sensation pénètre

Toutes les chairs, puis les os et les moelles enfin,

Qu'il s'agisse du plaisir ou d'une ardeur contraire ${ }^{58}$.

Les propriétés de la quatrième nature lui permettent de réagir la première aux chocs des simulacres. Une fois la quatrième nature mise en mouvement, elle frappe l'élément le plus fin après elle, la chaleur, qui à son tour frappe le souffle. Le dernier élément de l'âme à être frappé par ce mouvement de propagation graduelle est l'air, qui transmet ensuite le choc à la matière corporelle la plus mobile, à savoir le sang. Après cela, tout le corps, petit à petit, est mis en mouvement par cette chaîne de chocs perceptifs, comme dans un effet domino, qui a son origine dans la matière la plus fine de l'âme et qui s'achève dans la matière la plus « lourde » et la plus stable du corps, les os ${ }^{59}$.

Il faut désormais vérifier le degré de proximité entre le témoignage de Cicéron et les vers de Lucrèce, en observant la structure argumentative et le lexique de ces deux passages. Dans le passage transmis par Cicéron, le raisonnement est le suivant :

recoins les plus petits de l'agrégat corporel. À propos de la quatrième nature et de sa vitesse, voir Macé 2003, p. 119-165.

58. DRN III, v. 245-251.

59. En réalité la dernière matière à être touchée est la moelle osseuse, puisqu'elle se trouve à l'intérieur des os qui sont la matière la plus « lourde » et compacte. Cependant seule une sensation très violente arrive à propager les sensiferi motus jusque-là (v. 250). 
(1) Tous les êtres procèdent des quatre éléments terre, air, eau, feu ;

(2) l'âme est un être ;

(3) l'âme a les fonctions d'imaginer, prévoir, apprendre, enseigner, inventer, se souvenir, aimer et haïr, désirer et craindre, s'affliger et se réjouir ;

mais

(4) les éléments terre, air, eau et feu ne rendent pas raison de ces fonctions ; donc donc

(5) l'âme ne peut pas procéder des quatre éléments terre, air, eau et feu ;

(6) l'âme doit procéder d'un cinquième élément sans nom.

Le raisonnement semble très simple : si les quatre éléments qui constituent tous les êtres ne peuvent pas rendre compte des activités de l'âme, alors l'âme ne peut pas être constituée par ces quatre éléments, mais il faut déduire l'existence d'un cinquième. Cet élément est introduit parce qu'est reconnue l'incapacité des quatre éléments à rendre compte d'une activité. L'introduction de la quintessence se fait donc par un argument par soustraction. Examinons la structure argumentative utilisée par Lucrèce pour introduire la quatrième nature :

(1) Le dernier acte d'un corps vivant qui est sur le point de mourir est le dernier souffle ;

(2) la mort est le départ de l'âme ; donc

(3) le dernier souffle coïncide avec l'âme ;

or donc

(4) le souffle est composé d'aer, ventus et calor; mais

(5) L'âme est composée d'aer, ventus et calor;

(6) aer, ventus et calor ne peuvent pas rendre compte des activités de donc l'âme(pensée et sensation) ;

(7) il faut qu'il y ait un autre élément qui rende compte de ces activités ;

(8) cet élément est une quatrième nature sans nom.

Pour ce qui concerne les objets de ces raisonnements, il y a évidemment plusieurs différences. Premièrement, les deux théories ne se réfèrent pas au même type d'éléments : d'une part, on a les quatre éléments classiques, 
air-feu-eau-terre ${ }^{60}$; d'autre part, on trouve le triptyque air-vent-chaleur, trois natures tirées de l'expérience, c'est-à-dire de l'observation d'un homme mourant. L'autre différence importante réside dans le fait que dans le passage cicéronien le quintum genus est alternatif aux quatre autres natures et s'avère être le seul à constituer l'âme. Au contraire, la quarta natura épicurienne s'ajoute aux trois autres dans la composition de l'âme. Cela implique une autre différence : pour l'Aristote de Cicéron, l'âme est pure et non mélangée, ce qui ne correspond pas du tout à la conception épicurienne.

Une autre différence consiste évidemment dans le fait que la quatrième nature n'est pas un élément qui constitue les astres ni une nature propre aux dieux. Dans les premiers, ne se trouve pas d'âme, puisque le siège de chaque chose est déterminé ${ }^{61}$. Les dieux quant à eux sont doués d'une constitution qui leur est propre et qui justifie leur immortalité ${ }^{62}$. Or, puisque les hommes sont entièrement mortels, corps et âme, cette constitution divine ne peut leur appartenir et ne peut donc pas être identifiée à la quatrième nature.

Malgré ces différences entre les deux systèmes, il y a au moins deux aspects par lesquels les théories présentent des similarités. Observons d'abord le lexique. Des périphrases qui témoignent de la difficulté de nommer la nature de l'âme se retrouvent chez les deux auteurs : quintum genus vacans nomine chez l'un et quarta natura omnino nominis expers chez l'autre ${ }^{63}$. Ces périphrases se retrouvent chez les doxographes qui aident à reconstituer les théo-

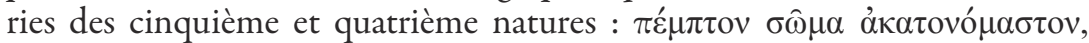

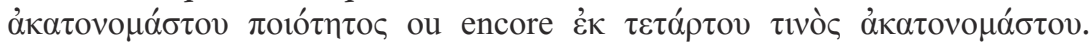
L'autre aspect très significatif est la structure argumentative. Tant chez Lucrèce que chez Cicéron, l'argument par soustraction paraît suivre le même chemin. La reconnaissance de l'incapacité de certaines natures communes à rendre compte - et donc à produire - des activités proprement psychologiques et consécutivement le recours à une nature supplémentaire caractérisent les deux passages.

La quintessence chez Cicéron et la quatrième nature chez Lucrèce n'ont pas le même rôle, puisqu'elles ont des caractéristiques différentes, et leur introduction vise des objectifs différents : dans le premier cas, il s'agit d'un élément divin, pur, non mélangé, alternatif à tous les autres ; dans

60. Sur la différence de conception des éléments entre Aristote et Épicure, voir Bénatouil 2003, p. 18-19.

61. DRN III, v. 131.

62. $D R N$ V, v. 146-155, 1211-1217; Ep. Men. 123-124.

63. L'« innommabilité » de la quatrième nature pourrait trouver une explication dans la conception empiriste du langage défendue probablement par Épicure. Comme l'écrit justement Annas 1992, p. 139: « Epicurus is hardly reluctant to coin new jargon elsewhere. Here he is constrained by his empiricism about concepts and language [...] In the case of the fourth kind of atom there is nothing in our experience capable of giving us any, even partial, idea of what it is like. » 
le deuxième cas, il s'agit d'un composé atomique, qui se mélange avec les autres natures. Cependant, elles jouent le même rôle par rapport aux autres éléments à l'intérieur du système auquel chacune appartient. Comment peut-on expliquer cette ressemblance frappante ?

Comme on l'a vu, la théorie de la quintessence pourrait appartenir au premier Aristote, dont les ouvrages publiés, selon le témoignage cicéronien, circulaient encore à l'époque de Cicéron et de Lucrèce. Cependant, les spécialistes sont souvent sceptiques à propos de cette hypothèse. L'autre possibilité, en suivant le témoignage de Jamblique et en le croisant avec les informations fournies par les doxographes, est que cette théorie soit à attribuer à des péripatéticiens d'orientation matérialiste comme Critolaos et Diodore de Tyr, dont l'activité remonte au $\mathrm{II}^{\mathrm{e}}$ siècle av. J.-C. Dans les deux cas, Cicéron tout comme Lucrèce pourraient l'avoir connue, et Lucrèce en particulier pourrait s'en être inspiré et s'être approprié l'argumentation par soustraction sur laquelle est fondée sa théorie. La proximité frappante entre les deux théories pour ce qui concerne la structure argumentative pourrait ainsi s'expliquer.

\section{Aristote, Épicure et l'argument par soustraction}

Toutefois, attribuer la théorie de la quintessence au Péripatos du II ${ }^{\mathrm{e}}$ siècle av. J.-C. et expliquer la ressemblance entre les structures argumentatives qui caractérisent le témoignage cicéronien et l'exposé lucrétien par la seule influence de cette théorie sur Cicéron et Lucrèce, impliquerait d'attribuer la conception de la quarta natura au seul Lucrèce. Épicure ne serait-il dès lors pour rien dans cette théorie de la quatrième nature ? Si le poème de Lucrèce constitue assurément une des sources les plus importantes pour les études épicuriennes et à plusieurs titres ${ }^{64}$, des doutes ont parfois été soulevés à propos de la fidélité de Lucrèce à son maitre et donc sur la fiabilité des informations que le poète latin nous a transmises. Les deux perspectives ${ }^{65}$ les plus célèbres sur cette question sont, sans aucun doute, celle de Piet Schrijvers, selon lequel Lucrèce était un philosophe éclectique activement engagé dans les débats philosophiques de son époque ${ }^{66}$ et, à l'extrême opposé, celle de David Sedley selon lequel le poète aurait été un épicurien « fondamentaliste » suivant de

64. Premièrement parce qu'il nous permet d'avoir accès à toute une série de doctrines exposées dans les ouvrages d'Épicure perdus, deuxièmement parce qu'il donne à voir l'épicurisme tel qu'il était dans un contexte assez éloigné, géographiquement et chronologiquement, d'Épicure et de son activité ; troisièmement parce que l'œuvre témoigne d'un effort d'originalité stylistique puisqu'il s'agit d'un poème, une forme normalement étrangère aux méthodes et aux moyens de communication de la doctrine des épicuriens de la première et de la deuxième génération, même si Philodème était aussi un poète. À propos de l'activité poétique de ce dernier, voir Gigante 1988. 2012.

65. Pour d'autres renseignements sur la question, je renvoie à l'étude récente de Montarese

66. Schrijvers 1999, p. 167-182. 
près le traité De la nature d'Épicure ${ }^{67}$.

L'hypothèse selon laquelle la conception de la quarta natura serait à attribuer à Lucrèce ne poserait aucun problème à ceux qui, comme Schrijvers, voient en Lucrèce un philosophe « indépendant ». Dans ce cas-là, Lucrèce aurait pu développer la théorie de la quarta natura à partir de la psychologie atomiste d'Épicure et sous l'influence de la théorie de la quintessence issue du Péripatos, assez connue à son époque, comme le rappelle le témoignage de Cicéron. Il pourrait donc s'en être inspiré et s'être approprié l'argument par soustraction qui est au cœur de la théorie. Mais la conception de la quarta natura et sa justification ne pourrait-elle remonter à Épicure ? Un témoignage d'Aëtius déjà mentionné le laisse entendre ${ }^{68}$. Épicure ne pourrait-il pas lui-même avoir produit un tel argument à partir d'une source péripatéticienne?

À l'intérieur du corpus aristotélicien, deux passages présentent un argument par soustraction qui pourrait rappeler celui qui caractérise le témoignage de Cicéron et les vers de Lucrèce : il s'agit du passage du De Caelo (269a30-269b2) dans lequel Aristote introduit l'éther, et du passage de La Génération des animaux (736b29-737a1) dans lequel Aristote explique comment l'âme est présente dans le sperme. Dans le De Caelo, l'argument par soustraction est utilisé par Aristote pour introduire l'éther comme élément doué de mouvement circulaire. Le schéma a été ainsi formalisé par R. J. Hankinson :

(1) All motion is either natural or unnatural (but not both);

(2) for any $\mathrm{y}$, if $\mathrm{m}$ is unnatural for $\mathrm{y}$, then there is an $\mathrm{x}$ such that $\mathrm{m}$ is natural for $\mathrm{x}$; bence,

(3) if circular motion is unnatural for earth, water, air and fire, there is an $\mathrm{x}$ such that circular motion is natural for $\mathrm{x}$;

(4) the natural motions of earth and water are downward, of fire and air upward; bence,

(5) every body has at most one natural motion;

(6) circular motion is not natural for earth, water, air and fire; bence,

(7) circular motion is unnatural for earth, water, air and fire; bence,

(8) there is an $\mathrm{x}$, distinct from earth, water, air and fire, such that circular motion is natural for $\mathrm{x}$;

And $x$ of course is the aether ${ }^{69}$.

67. Sedley 1998, p. 71-72.

68. Aëtius $4.3 .11=$ Us. 315 .

69. Hankinson 2009, p. 103-104. 
La clé de ce raisonnement est la théorie des lieux naturels d'Aristote qui établit que chaque élément possède un lieu auquel il appartient naturellement et vers lequel il aura tendance à revenir ${ }^{70}$. Dans cette tendance consiste le mouvement naturel de chaque élément auquel correspond toujours un mouvement non naturel et vice versa. Sur la base de la non-naturalité du mouvement circulaire pour la terre, l'eau, l'air et le feu, Aristote établit la nécessité de l'existence d'un élément pour lequel ce mouvement soit naturel. Cet élément est justement l'éther. L'introduction de l'éther est justifiée par la nécessité d'identifier un élément doué d'un mouvement qui n'appartient pas naturellement aux autres éléments. Autrement dit, l'introduction de l'éther répond à l'impossibilité pour les autres natures de rendre raison du mouvement circulaire des astres. La théorie des lieux est l'« outil » argumentatif employé par Aristote. Bien que la structure argumentative qui caractérise le passage d'Aristote soit plus complexe que celles de Cicéron et de Lucrèce, puisqu'elle implique la théorie des lieux, elle est tout de même remarquablement proche de celles des deux auteurs latins. Encore une fois, l'élément supplémentaire, en l'occurrence l'éther, n'a pas la même fonction que les éléments supplémentaires dont parlent Cicéron et Lucrèce. En effet, le contexte dans lequel l'introduction de l'éther s'inscrit ici est un contexte cosmologique, et non pas psychologique, et la caractéristique principale pour laquelle il est introduit est le mouvement circulaire dont il est « naturellement » doué. Cependant, l'éther joue par rapport aux autres éléments le même rôle que, respectivement, la quintessence et la quarta natura par rapport aux autres éléments en complément desquels elles sont introduites.

Passons au deuxième livre de La Génération des animaux:

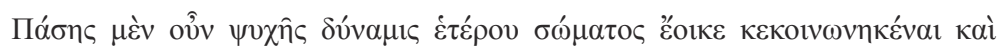

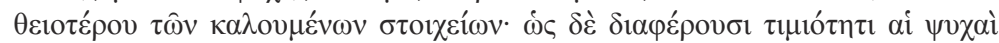

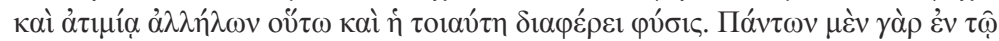

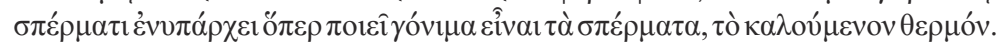

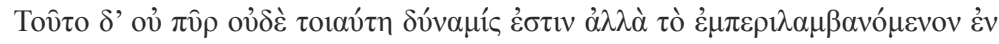

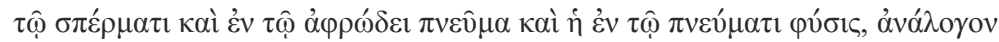

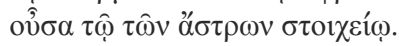

La puissance de chaque âme semble donc avoir participé d'un corps différent et plus divin que ceux qu'on appelle les éléments. Comme les âmes different entre elles par la valeur et par l'absence de valeur, c'est de la même façon que differe une telle nature. En effet, dans le sperme de tous existe ce qui précisément rend fécondes les semences, ce qu'on appelle le chaud. Cela n'est ni le feu ni une puissance du même genre, mais le pneuma qui est enfermé dans le sperme écumeux et c'est la nature [enfermée] dans le pneuma qui est analogue à l'élément des astres ${ }^{71}$.

70. Cael.269a30-269b2.

71. GA II, 3, 736b29-737al. Texte établi par Drossaart Lulofs 1965 ; ma traduction. 
Cet extrait est le passage conclusif d'une argumentation très serrée qui va de la ligne 736a29 à la ligne 737a15 et qui s'interroge sur l'âme par laquelle l'animal se définit (l'âme sensitive, donc) : est-elle présente dans le sperme ou dans le produit de la conception, c'est-à-dire l'embryon ? Et dans ce cas, d'où provient-elle ? Avant d'examiner ce passage, on peut résumer les étapes précédentes de l'argumentation. Aristote, après avoir présenté le problème à résoudre, exclut immédiatement que le sperme et le produit de la conception puissent être inanimés. Il affirme qu'ils ne sont pas moins doués de vie que les plantes. Le philosophe reconnaît donc au sperme et au produit de la conception un premier « degré » de vie qui correspond à l'âme végétative ou nutritive. Or, puisqu'ils possèdent l'âme végétative, en se développant, ils possèdent aussi l'âme sensitive qui est celle qui fait d'un être vivant un animal. Cela arrive dans un deuxième moment puisqu' « on ne devient pas simultanément animal et homme ni animal et cheval », étant donné que l'accomplissement d'un être arrive à la fin. La même chose se produira évidemment pour l'âme intellective. Or, pour que cela soit possible, il faut admettre que l'âme se trouve dans les êtres en puissance ${ }^{72}$ et qu'elle s'actualise une fois exercée l'activité dont elle est principe (dans le cas de l'âme végétative, par exemple, la nutrition). Mais comment est-il possible que ces âmes soient déjà dans le sperme ? Aristote exclut tout de suite la possibilité que ces âmes soient préexistantes et entrent de l'extérieur dans le sperme : puisqu'elles sont principes d'activités corporelles, elles ne peuvent pas exister sans un corps. De la même façon, il exclut qu'elles puissent entrer dans le corps ensuite, parce qu'elles ne peuvent pas exister sans un corps et parce que le sperme se forme à partir de la transformation de la nourriture, donc à l'intérieur du corps. Cependant, ces raisonnements ne sont valables que pour l'âme végétative et sensitive, étant donné que l'âme intellectuelle n'est pas principe d'une activité corporelle et pourrait donc provenir de l'extérieur.

C'est à ce moment précis que se trouve notre passage qui, comme on le verra, n'est pas la conclusion de l'argumentation mais seulement une considération conclusive relative à l'argument de la préexistence des âmes et de leur origine externe ${ }^{73}$. Aristote, en effet, poursuit son discours sur l'âme intellective, qu'il avait décrite un peu avant comme divine, en distinguant son activité de celle du corps et en affirmant que cette faculté de l'âme semble participer d'un corps différent et plus divin que ceux qui composent le monde, à savoir l'air, l'eau, la terre et le feu. Le raisonnement est le suivant : toutes choses se distinguent entre elles selon les valeurs qu'elles possèdent ; de la même façon, l'âme intellective ou le corps dont elle participe se distingue par une certaine valeur par rapport aux quatre éléments. Mais quel est ce corps ? Le philosophe affirme qu'il s'agit d'une certaine chaleur qui se trouve dans

72. À ce propos, voir aussi Code 1987, p. 51-59.

73. À ce propos, voir Berti 2010, p. 494-498. 
le sperme, qui rend la semence féconde. Cette chaleur n'appartient pas à la nature du feu ni à autre chose de semblable mais à une nature différente : le pneuma. Cette substance, selon le philosophe, est enfermée dans le sperme écumeux $^{74}$. La nature contenue dans le souffle est analogue, dit-il, à l'élément qui constitue les astres, à savoir l'éther.

Les éléments qui sont mis en relation dans ce passage sont ainsi au nombre de trois : la chaleur, le pneuma et l'éther. Pour ce qui concerne la chaleur et le pneuma, je ne traiterai pas ici dans le détail de tous les emplois aristotéliciens de ces concepts mais je me limiterai à résumer leurs significations principales. Il semble exister deux types de chaleur chez le Stagirite : une chaleur qui dérive du feu et une chaleur pneumatique. La chaleur du pneuma et celle du soleil sont distinguées de la chaleur du feu. En effet, si la nature du feu ne fait pas naître les animaux, la chaleur du pneuma et celle du soleil

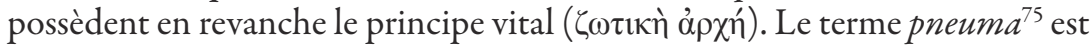
utilisé par Aristote dans plusieurs sens. Le plus simple est sans doute celui de « souffle », à l'occasion de l'explication du mécanisme respiratoire, mais Aristote l'emploie aussi pour désigner le principe vital interne à l'être vivant qui permet le mouvement (dans Le Mouvement des animaux) et le principe de vie (dans La Génération des animaux). Or, les rapports qui lient ces trois significations du pneuma chez le philosophe doivent être cherchés à mon avis du côté de l'analogie avec l'éther. En effet, Aristote affirme que l'âme intellective est trop divine pour que sa nature s'explique à partir des quatre éléments, et qu'elle semble par ses caractéristiques participer d'un corps divin. Cela s'explique aussi par le fait que l'âme intellective n'a pas la même relation que les autres âmes avec le corps. Autrement dit, l'âme intellective, n'étant pas le principe de fonctions du corps, n'a rien à voir avec les éléments dont le corps est composé. L'âme intellective doit par conséquent participer d'une nature alternative et cette nature est la chaleur du pneuma. Ce raisonnement suit le même schéma argumentatif par soustraction que celui du De Caelo : puisque les quatre éléments ne peuvent pas rendre compte du mouvement circulaire des astres parce qu'aucun d'eux n'en est doué naturellement, il faut admettre l'existence d'un cinquième élément. De la même façon, puisque les quatre éléments ne rendent pas compte de l'activité (divine) de l'âme intellective,

74. Ailleurs ( $G A$ II, 2, 735b21-22), Aristote affirme que le sperme est une substance qui ressemble à l'écume (ג’ $\varphi$ ó́). Le rapport entre sperme et écume se trouve déjà chez Diogène

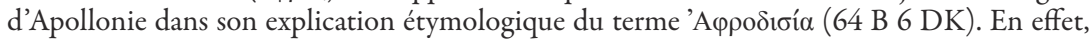
dans le récit d'Hésiode, Aphrodite serait née de l'écume de la mer quand Chronos y avait jeté le membre d'Ouranos après l'avoir émasculé (Théogonie, v. 185-206). On trouve aussi des représentations de ce type chez les médecins dits hippocratiques, notamment dans le traité $D e$ la Génération, dans la description de l'éjaculation des chapitres 1 et 2.

75. Pour un approfondissement de cette question, voir Jaeger 1913, p. 29-74; Wiersma 1943, p. 102-107 ; Peck 1953, p. 111-121 ; Solmsen 1957, p. 119-123 ; Verbeke 1978, p. 191-214; Freudenthal 1995 ; Bos 2006, p. 35-72. 
il faut admettre une cinquième nature capable de le faire. Ainsi, la nature du pneuma est un óvód oyov de l'éther, dans le sens où elle est, par rapport aux autres éléments du composé biologique de La Génération des animaux, ce que l'éther est aux autres éléments cosmologiques dans le De Caelo. L'analogie entre les deux natures ne se base donc pas sur une proximité entre les deux matières ou entre leurs qualités propres, mais sur la façon dont Aristote les a identifiées ${ }^{76}$.

Ainsi, l'argument par soustraction qui caractérise l'introduction de la quintessence chez Cicéron existe déjà dans le corpus aristotélicien avant Critolaos et Diodore de Tyr et, de plus, à propos de deux sujets proches du témoignage cicéronien, l'éther d'une part, et l'éther et l'âme/pneuma de l'autre. Il est donc possible que la théorie que rapporte Cicéron ait été développée par les péripatéticiens à partir de ce genre d'arguments aristotéliciens. Si cela est vrai, il n'est pas nécessaire d'attribuer la conception de la théorie de la quarta natura à Lucrèce afin d'expliquer l'appropriation épicurienne de l'argument par soustraction. En effet, puisqu'il s'agit d'un argument aristotélicien, il est possible qu'Épicure en ait déjà eu connaissance et qu'il s'en soit servi dans sa théorie psychologique.

Or, comme on l'a dit en commençant, Boyancé a exclu la possibilité d'un quelconque rapport d'influence entre la théorie de la quintessence exposée par Cicéron et la théorie de la quatrième nature exposée par Lucrèce, parce que « ce qui importe pour juger une doctrine comme celle-là, ce n'en est pas tant la lettre, que l'orientation de la pensée qui la formule. Cette orientation est vers le matérialisme, non vers le spiritualisme ». Cette affirmation semble valoir comme objection non seulement à la proposition d'une influence de la théorie de la quintessence sur Lucrèce mais aussi à l'hypothèse qu'on est en train d'examiner, de l'influence d'un argument aristotélicien sur Épicure. En effet, les systèmes aristotélicien et épicurien sont entièrement différents comme sont différentes leurs « orientations ». Mais sommes-nous sûrs qu'aucune forme d'appropriation d'un concept ou d'un argument ne peut avoir lieu si les objectifs des systèmes impliqués n'ont pas la même « orientation »? Et surtout est-ce vrai dans le cas des philosophies du Jardin et du Péripatos?

Cette question est en fait au cœur des débats sur le rapport entre ces deux écoles philosophiques ${ }^{77}$. Gassendi ${ }^{78}$, dans ses études sur l'œuvre d'Épicure, avait déjà décelé des liens entre certains éléments de la doctrine d'Épicure

76. La chaleur du pneuma n'est pas un élément comme l'est en revanche l'éther.

77. Pour un regard approfondi sur le rapport entre les deux écoles, voir Furley 1967 ; Angeli 1988, p. 236-237 ; De Lacy \& De Lacy 1978, p. 175 n. 41 ; Gigante 1999 ; Sedley 1976, p. 126-127 ; Verde 2013, p. 3-9; Verde 2016, p. 35-55.

78. Cf. Alberti 1988, p. 93 sq. 
et la tradition aristotélicienne. Bailey ${ }^{79}$ a reconnu des traces aristotéliciennes chez Épicure, mais il excluait toute intention polémique, et Diano ${ }^{80}$ a montré les dettes philosophiques d'Épicure à l'égard de la psychologie d'Aristote. Cependant, les deux études les plus célèbres des interactions entre les deux doctrines sont sans aucun doute L'Aristotele perduto e la formazione filosofica di Epicuro ${ }^{81}$ d'Ettore Bignone et Two Studies in the Greek Atomists ${ }^{82}$ de David Furley. Le premier a développé la thèse selon laquelle la formation philosophique d'Épicure avait ses racines dans la connaissance de l'Aristote des dialogues, aujourd'hui perdus, et que les racines de sa pensée seraient à chercher justement dans la confrontation avec l'Aristote des écrits soi-disant exotériques. De plus, Bignone excluait complètement la possibilité qu'Épicure ait eu accès aux écrits ésotériques qui nous ont été transmis. Cette position a été largement discutée et est encore défendue par certains ${ }^{83}$, mais la plupart des savants ${ }^{84}$ sont aujourd'hui d'accord sur le fait qu'Épicure a subi l'influence de la pensée aristotélicienne des écrits non publiés autant que des dialogues. Au-delà des résultats de L'Aristotele perduto, l'étude de Bignone a sans aucun doute le mérite d'avoir inauguré une nouvelle orientation de la recherche dont probablement le meilleur produit est l'étude de Furley. Contrairement à Bignone, qui avait essayé de reconstituer la phase platonicienne des études d'Aristote, en spéculant sur l'impact de cette dernière sur le jeune Épicure, Furley étudie parallèlement les écrits d'Aristote et ceux d'Épicure et met en évidence des correspondances intéressantes autant dans le domaine physique qu'en éthique. Son Two Studies in the Greek Atomists examine en particulier deux sujets, la théorie des minima et la théorie de la libre volonté, avec une même approche : comprendre les deux théories en les considérant comme la réponse d'Épicure à des questions déjà soulevées par Aristote. Dans le premier cas, Furley analyse la théorie des parties minimales (et indivisibles) qui composent l'atome en la mettant en relation avec la critique d'Aristote dans Physique IV à l'égard des atomes indivisibles (physiquement et théoriquement) de Démocrite. Dans le deuxième cas, Furley explique la conception épicurienne de la libera voluntas et la théorie du clinamen à la lumière de l'Éthique à Nicomaque. Le résultat le plus important de ces études est une nouvelle conception du rapport d'Épicure à Aristote, une conception, pour ainsi dire, dialectique ${ }^{85}$. Cette thèse trouve une

79. Bailey 1928, p. 24.

80. Diano 1939-1942.

81. Bignone $1973^{2}$.

82. Furley 1967.

83. Pesce 1974.

84. Outre ceux cités supra n. 77, on mentionnera Jannone 1996, p. 87-98 ; Montarese 2012, p. 140-143; Zemplen 2005, p. 102.

85. À ce propos, voir Verde 2016, p. 36. 
confirmation dans un témoignage très précieux de Simplicius ${ }^{86}$ où le philosophe affirme qu'Épicure aurait partagé la pensée de Démocrite mais était bien conscient des critiques qu'Aristote lui avait adressées. Comme l'écrit Graziano Arrighetti : «L'atomismo di Epicuro si rifá, si, a quello democriteo, ma tramite Aristotele; cioè tenendo presenti tutte le critiche dello Stagirita a quel sistema, tutte le nuove istanze da lui messe in luce ${ }^{87}$. > Les résultats de ces études sur le rapport dialectique entre Aristote et Épicure ont été confirmés par des études plus récentes comme celle de Francesco Verde ${ }^{88}$, où le chercheur propose une reconstitution de la théorie des minima à partir du contexte philosophique antérieur et contemporain d'Épicure et se concentre notamment sur l'influence aristotélicienne.

À la lumière des résultats obtenus par ces spécialistes, il est légitime d'envisager qu'Épicure ait pu s'approprier l'argument par soustraction aristotélicien et ce, malgré la mise en garde de Boyancé sur la différence d'orientation des deux systèmes. Épicure ne pourrait-il pas même s'être approprié l'argument d'Aristote afin de répondre de façon plus efficace à une critique aristotélicienne ayant pour cible la théorie matérialiste démocritéenne de la composition de l'âme?

Selon la conception démocritéenne, dont Aristote est un des témoins principaux, l'âme est un corps composé d'atomes de forme sphérique, identiques à ceux qui constituent le feu et la chaleur ${ }^{89}$. Selon le philosophe de Stagire, cela s'expliquerait par la tentative de la part de Démocrite de rendre raison de la capacité motrice de l'âme. En effet, la conception démocritéenne de l'âme semble identifier dans l'auto-motricité le caractère qui distingue les êtres animés des êtres non animés. Afin de rendre raison de ce caractère propre à l'âme, il aurait choisi parmi les atomes existants ceux qui constituent aussi le feu. Voici un passage du De anima, où Aristote attribue cette idée à d'autres philosophes ${ }^{90}$, outre Démocrite :

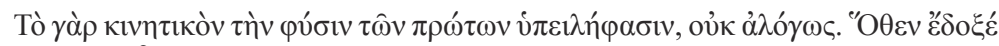

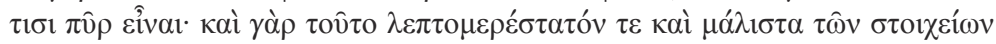

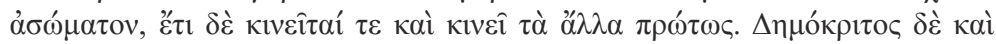

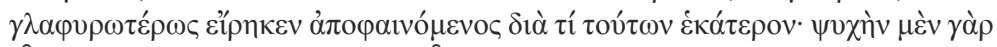

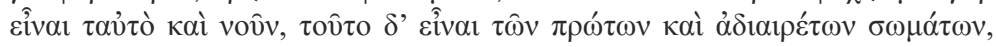

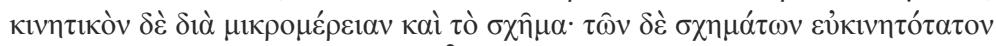
$\tau$

Ils ont, en effet, pris pour acquis, non sans raison, que la réalité de nature motrice fait partie des réalités primordiales. D'où l'opinion de certains, pour

86. In Phys. 925.13 Diels $=268$ Us.

87. Arrighetti 1960, p. XXXII $s q$.

88. Verde 2013.

89. De An. I, 2, 403b30-404a15.

90. Probablement Héraclite, selon Bodéüs 1993, p. 96 n. 3. 
qui cette réalité est le feu. Car ce dernier constitue à la fois le plus subtil et le moins corporel des éléments. Et, de surcroît, sa mobilité, ainsi que sa motricité à l'égard de tout le reste, sont primordiales. Démocrite a d'ailleurs prétendu, dans un exposé très brillant, fournir la raison de ces deux caractères. L'âme, en effet, serait la même chose que l'intelligence, cette chose figurerait parmi les corps primordiaux et indivisibles et elle devrait sa propriété motrice à sa subtilité et à sa configuration. Il affirme par ailleurs que, d'entre les figures, la plus aisément mobile est celle de la sphère. Tels sont précisément l'intellect et le feu ${ }^{91}$.

Selon Aristote ${ }^{92}$, Démocrite aurait donc identifié les composants de l'âme aux atomes sphériques qui constituent aussi le feu et qui, à cause de leur forme, peuvent expliquer à la fois la mobilité de l'âme et sa capacité motrice à l'égard du corps. En ce sens, Démocrite aurait choisi le feu qui est doué à la fois de mobilité et de motricité plutôt que les autres éléments, doués de la première caractéristique mais non de la seconde. Cependant, un élément du compte-rendu aristotélicien mérite une attention toute particulière. En effet, Aristote affirme que ceux qui ont identifié l'âme avec le feu (ou avec ses composants comme Démocrite) l'ont fait parce que celui-ci est à la fois

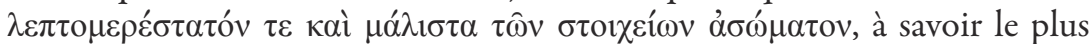
subtil et le moins corporel des éléments. Or, affirmer qu'un atomiste identifie dans le feu (et ses composants) la matière constitutive de l'âme parce qu'il s'agit de l'élément le moins corporel semble sonner comme un reproche ou une critique. Aristote veut signaler que le problème du mouvement de l'âme pousse l'atomiste dans ses retranchements ou plutôt jusqu'à ses dernières extrémités, puisque le corps qu'il invoque pour rendre compte de l'âme n'a presque plus aucune caractéristique des corps perceptibles. Aristote veut voir là un aveu des limites, voire de l'incohérence de l'atomisme, de son incapacité d'expliquer l'âme, et donc tous les vivants.

Il y a des ressemblances entre cette analyse aristotélicienne de la position démocritéenne et les vers de Lucrèce sur la quatrième nature, tout comme avec les témoignages d'Aëtius ${ }^{93}$ et de Plutarque ${ }^{94}$ sur la quatrième nature chez

91. De An. I, 2, 405a4-13. Trad. Bodéüs 1993 légèrement modifiée.

92. Sur Aristote témoin de Démocrite, voir Morel 1996 et Sassi 1978.

93. Aëtius, 4.3.11 = 315 Us.

94. Plutarque, $A d v$. Col. 20, p. 1118 D. Dans ce passage, Plutarque explique et critique la théorie de la quatrième nature. Cette critique s'inscrit dans le cadre de la défense de Socrate contre les accusations de Colotès à propos des enquêtes sur ce qu'est un homme. L'objectif est de montrer l'incohérence de la psychologie d'Épicure qui théorise une âme matérielle, composée d'éléments matériels, mais qui est obligée, pour rendre raison des activités propres à l'esprit, d'introduire un autre élément, toujours matériel, mais propre aux êtres sensibles : un élément sans nom. Le fait de ne pas donner de nom à cette nature est interprété par Plutarque comme un aveu d'ignorance du philosophe et de ses adeptes. Par sa critique, il souligne donc les aspects les plus complexes et, d'un certain point de vue, les plus faibles, d'une théorie qui essaie de rendre compte des activités psychiques par le seul moyen de la matière. 
Épicure. En effet, Lucrèce affirme que les natures qui constituent l'âme, à savoir la chaleur, le souffle et l'air, ne peuvent pas expliquer la perception et la pensée. La raison en réside dans le fait que ces deux activités, propres à l'âme, qui s'expliquent en termes de mouvement, ne peuvent pas être accomplies par ces natures qui ne sont pas suffisamment subtiles donc suffisamment sensibles au mouvement. Dans la doctrine épicurienne aussi bien que dans le témoignage aristotélicien sur Démocrite, les activités propres à l'âme s'expliquent par une capacité cinétique exceptionnelle (par rapport aux autres corps), capacité elle-même expliquée à l'intérieur du cadre matérialiste en recourant à une matière exceptionnellement subtile. Cette matière, dans la théorie épicurienne, est la quatrième nature. En ce sens, Lucrèce assume l'analyse (explicite) d'Aristote, qui exige d'aller aux confins de l'atomisme pour rendre compte de l'exceptionnalité de l'âme, mais non sa conséquence implicite pour Aristote, à savoir l'impossibilité d'atteindre cet objectif dans les limites de l'atomisme.

L'appropriation de l'argument aristotélicien par soustraction a pu représenter pour Épicure une façon de résoudre le problème de l'exceptionnalité cinétique de l'âme en dépassant la solution démocritéenne. En effet, comme on l'a vu dans les vers de Lucrèce, Épicure ne considère pas les atomes qui constituent le feu et la chaleur comme assez fins pour répondre aux stimulations perceptives. Il est probable que cela dérive du fait, communément observable, que tous les êtres doués de chaleur ne sont pas doués de sensation. Il est donc possible qu'Épicure ait trouvé dans l'argument par soustraction une façon de sortir de cette impasse en répondant à l'exigence de rendre compte de l'exceptionnalité de l'âme et de ses activités, sans pour autant remettre en cause l'atomisme. En effet, l'appropriation de la stratégie argumentative aristotélicienne par Épicure représente la renonciation du philosophe du Jardin à chercher l'élément proprement psychique parmi les natures connues, au profit de l'identification d'une nature supplémentaire inconnue. L'absence de nom de cette quatrième nature signale notamment la volonté d'Épicure d'introduire une matière nouvelle qui ne ressemble pas à celles déjà proposées par ses prédécesseurs. Au lieu donc d'essayer de démontrer comment une nature connue et commune peut devenir la condition d'activités exceptionnelles comme la sensation, à l'instar de Démocrite, Épicure déduit l'existence de la nature psychique de l'impossibilité que les autres natures rendent compte de ce genre d'activités. Utiliser l'argument présent dans le De caelo et dans le De generatione en l'adaptant au cadre atomiste aurait ainsi conduit Épicure à résoudre le problème de l'exceptionnalité de l'âme en mobilisant un élément matériel supplémentaire capable d'expliquer des phénomènes évidents, comme la perception et la pensée, que les autres natures ne peuvent pas expliquer. En effet, comme pour l'éther et le pneuma, la quatrième nature est une matière dont on n'a pas une connais- 
sance empirique immédiate mais dont l'existence est déduite logiquement. En ce sens, il s'agirait d'un autre cas de reprise dialectique, par Épicure, d'un élément appartenant à la philosophie aristotélicienne qui, une fois intégré par Épicure à sa doctrine, permet de répondre de façon plus efficace à une critique adressée par Aristote à la théorie atomiste de Démocrite.

\section{Conclusion}

On espère avoir rendu vraisemblable l'hypothèse selon laquelle la théorie de la quatrième nature peut s'expliquer par l'appropriation d'un argument aristotélicien par le Jardin. En effet, comme on l'a vu, la ressemblance dans la structure argumentative entre la théorie de la quintessence exposée par Cicéron et la théorie de la quarta natura exposée par Lucrèce est assez remarquable. Cette ressemblance pourrait s'expliquer par le fait que Cicéron et Lucrèce, qui sont contemporains, connaissaient la théorie de l'âme-éther qui, d'après ce que suggèrent les témoignages de Jamblique et des doxographes, aurait été développée par Critolaos et Diodore de Tyr, philosophes péripatéticiens dont l'activité se situe au $\mathrm{II}^{\mathrm{e}}$ siècle av. J.-C. Cette hypothèse, qui serait tout à fait légitime, impliquerait que la théorisation de la quatrième nature, ou au moins sa justification par soustraction, soit le fait du seul Lucrèce. Cependant, l'appropriation de l'argument par soustraction pour justifier la quatrième nature peut remonter à Épicure lui-même. En effet, l'argument par soustraction, comme celui grâce auquel la matière de l'âme est identifiée comme la quintessence, se trouve déjà chez Aristote dans le De Caelo et dans le De Generatione. Il est donc possible qu'Épicure se soit approprié l'argument aristotélicien en l'intégrant dans sa doctrine.

Un obstacle à cette interprétation a été identifié par Boyancé qui se montrait sceptique vis-à-vis de l'idée que deux systèmes aussi différents que ceux dans lesquels sont respectivement inscrites la quarta natura et la quintessence aient une influence l'un sur l'autre. Cette objection vaut évidemment aussi pour les systèmes épicurien et aristotélicien. Toutefois, des recherches récentes tendent à réévaluer le jeu de réappropriation dialectique d'Aristote par Épicure, qui malgré les différences d'《 orientation », aurait intégré des éléments aristotéliciens afin de renforcer certains points de la doctrine atomiste critiquée par ce même Aristote. Afin de vérifier si l'appropriation de l'argument par soustraction pourrait s'expliquer par la même démarche, j'ai analysé un passage du De Anima où Aristote semble identifier et critiquer la difficulté rencontrée et dépassée dans les vers de Lucrèce sur la quatrième nature : la nécessité d'aller au-delà des éléments matériels connus et de poser un élément exceptionnel pour expliquer les capacités de l'âme. L'appropriation par Épicure de l'argument par soustraction employé par Aristote pour introduire l'éther et le pneuma dans sa doctrine pourrait s'expliquer par la volonté de dépasser la difficulté d'identifier parmi les natures 
connues une matière capable d'expliquer les propriétés exceptionnelles de l'âme. En effet, puisque, selon Lucrèce, Épicure ne considère pas les atomes de chaleur comme assez fins et mobiles, mobilisant ainsi l'argument par soustraction d'Aristote, il résoudrait le problème de l'identification d'une matière proprement psychique en introduisant une nature supplémentaire douée des propriétés qui manquent aux natures connues. Épicure ferait donc de la quatrième nature l'analogue du pneuma et de l'éther, à savoir un élément supplémentaire dont l'existence est déduite à partir de certains phénomènes qui ne sont pas explicables autrement. Même si cette hypothèse finale d'un emprunt épicurien de l'argument par soustraction à Aristote demeure spéculative, il me semble que la comparaison qui la soutient éclaire la stratégie spécifique de la psychologie épicurienne par rapport à celle de Démocrite et à celle d'Aristote. 


\section{BIBLIOGRAPHIE}

Alberti, A. 1988 : Sensazione e realtà, Epicuro e Gassendi, Firenze, 1988 (Studi / Accademia toscana di scienze e lettere « La Colombaria », 95).

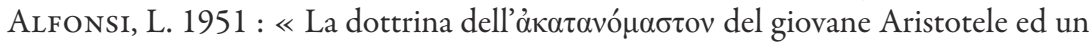
testo di Psello », Miscellanea Giovanni Galbiati, I, Archeologia, storia, filologia e antichità classica, papirologia, glottologia, arte, Milano, 1951, p. 71-78.

Angeli, A. 1988 (éd.) : Filodemo, Agli amici di scuola (PHerc 1005), Napoli, 1988 (La scuola di Epicuro, 7).

Annas, J. 1992 : Hellenistic philosophy of mind, Berkeley, 1992 (Hellenistic Culture and Society, 8).

Arrighetti, G. 1960 (éd.) : Epicuro, Opere, Torino, 1960 (Classici della filosofia, 4).

BAILEY, C. 1928 : The Greek Atomists and Epicurus, Oxford, 1928.

BÉNATOUÏL, T. 2003 : « La méthode épicurienne des explications multiples », dans T. Bénatouil, V. Laurand, A. Macé (éd.), L'Épicurisme antique, Les Cahiers philosophiques de Strasbourg, 15 (2003), p. 15-47.

Berti, E. 1962 : La filosofia del primo Aristotele, Padova-Milano, 1962 (Pubblicazioni della Facoltà di Lettere e Filosofia / Università di Padova, 38).

Berti, E. 2010 : « Natura e generazione degli animali in Aristotele », Kriterion, 51/122 (2010), p. 489-504.

Bignone, E. $1973^{2}$ : L'Aristotele perduto e la formazione filosofica di Epicuro, 2 vol., Firenze, $1973^{2}$ (Il Pensiero filosofico, 7, 1;7, 2).

Bodéüs, R. 1993 (trad.) : Aristote, De l'âme, Paris, 1993 (GF, 711).

Bos, A. P. 2006 : « The "Instrumental Body" of the Soul in Aristotle's Ethics and Biology », Elenchos, 27/1 (2006), p. 35-72.

BoyancÉ, P. 1958 : « La théorie de l'âme chez Lucrèce », Bulletin de l'Association Guillaume Budé, 17/Suppl. (1958), p. 30-49.

BRISSON, L. 2005 : «Epinomis : authenticity and authorship », dans K. Döring, M. Erler, S. Schorn (éd.), Pseudoplatonica. Akten des Kongresses vom 6-9 Juli 2003, Stuttgart, 2005, p. 9-24 (Philosophie der Antike, 22).

Brittain, C. 2006 (trad.) : Cicero, On Academic Scepticism, Indianapolis, 2006.

Brunschwig, J. \& P. Pellegrin 2001 (trad.) : A. A. Long \& D. N. Sedley (éd.), Les philosophes hellénistiques, 3 vol., Paris, 2001 (GF, 641, 642, 643).

Caston, V. 1997 : «Epiphenomenalisms, Ancient and Modern », The Philosophical Review, 106/3 (1997), p. 319-354.

Code A. 1987 : «Soul as Efficient Cause in Aristotle's Embryology », Philosophical Topics, $15 / 2$ (1987), p. 51-59.

De Lacy, P. H. \& E. A. De Lacy 1978 (trad.) : Philodemus, On Methods of Inference, Napoli, 1978 (La scuola di Epicuro, 1).

Della Valle, G. 1905 : « La teoria dell'anima-armonia di Aristosseno e l'epifenomenismo contemporaneo », Rivista filosofica, 7/2 (1905), p. 210-231.

Diano, C. 1939-1942 : «La psicologia d'Epicuro e la teoria delle passioni », Giornale critico della filosofia italiana, VII, VIII, IX, X (1939-1942), p. 105-145, p. 151-165, p. 5-34, p. 5-49 et p. 121-150 (= Scritti Epicurei, Firenze, 1974, p. 129-280). 
Drossaart Lulofs, H. J. 1965 (éd.) : Aristotelis de generatione animalium, Oxford, 1965 (Scriptorum classicorum bibliotheca Oxoniensis).

Festugière, A.-J. 1949 : La Révélation d'Hermès Trismégiste, II. Le Dieu cosmique, Paris, 1949.

Finamore, J. \& J. M. Dillon 2002 (éd.) : Iamblichus, De Anima, Text, Translation, and Commentary, Leiden-Boston-Köln, 2002 (Philosophia antiqua : a Series of Studies on Ancient Philosophy, 92).

Frerichs, H. 1892 : Quaestiones Lucretianae, Oldenburg, 1892 (Programm des Grossherzoglichen Gymnasiums zu Oldenburg).

Freudenthal, G. 1995 : Aristotle's Theory of Material Substance. Heat and Pneuma, Form and Soul, Oxford, 1995.

Furley, D. J. 1956 : « The Early History of the Concept of Soul », Bulletin of the Institute of Classical Studies, 3 (1956), p. 1-18.

Furley, D. J. 1967 : Two Studies in the Greek Atomists. I : Indivisible Magnitudes, II : Aristotle and Epicurus on Voluntary Action, Princeton, 1967.

Gigante, M. 1988 (éd.) : Filodemo, Epigrammi scelti, Napoli, 1988.

Gigante, M. 1999 : Kepos e Peripatos. Contributo alla storia dell' aristotelismo antico, Napoli, 1999 (Elenchos, 29).

Giussani, C. 1896 (éd.) : T. Lucreti Cari De Rerum Natura libri sex, revisione del testo, commento e studi introduttivi, Torino, 1896.

Gottschalk, H. B. 1971 : « Soul as Harmonia », Phronesis, 16/2 (1971), p. 179-198.

Hahm, D. E. 1982 : « The Fifth Element in Aristotle's De Philosophia : A Critical Re-Examination », The Journal of Hellenic Studies, 102 (1982), p. 60-74.

Hankinson, R. J. 2009 : « Natural, Unnatural, and Preternatural Motions : Contrariety and the Argument for the Elements in De caelo 1.2-4 », dans A. C. Bowen, C. Wildberg (éd.), New Perspectives on Aristotle's De caelo, LeidenBoston, 2009 (Philosophia antiqua, 117), p. 83-117.

Harward, J. 1928 (trad.) : The Epinomis of Plato, Oxford, 1928.

Humbert, J. 1931 (trad.) : Cicéron, Tusculanes, texte établi par G. Fohlen, Paris, 1931 (Collection des Universités de France).

Jaeger, W. 1913 : « Das Pneuma im Lykeion », Hermes, 48/1 (1913), p. 29-74.

Jannone, A. 1996 : « La polemica di Epicuro e Aristotele », dans G. Giannantoni et M. Gigante (éd.), Epicureismo greco e romano, Atti del Congresso Internazionale, Napoli, 19-26 maggio 1993, 3 vol., Napoli, 1996, vol. I (Elenchos, 25), p. 87-98.

JounnNa, J. 1987 : «L Le souffle, la vie et le froid. Remarques sur la famille de $\psi v 0$ d'Homère à Hippocrate », Revue des études grecques, 100/477-479 (1987), p. 203-224.

Kany-Turpin, J. 1993 (trad.) : Lucrèce, De la nature, Paris, 1993 (Bibliothèque philosophique bilingue).

Kerferd, G. B. 1971 : « Epicurus' Doctrine of the Soul », Phronesis, 16/1 (1971), p. $80-96$.

Konstan, D. 2007 : Lucrezio e la psicologia epicurea, trad. it I. Ramelli, Milano, 2007 (Studi e testi, 103).

LEONE, G. 2002 : « Nuove conferme dall'opera Della natura di Epicuro alla valenza 
tecnica del gruppo semantico hetoimos/hetoimôs nella dottrina epicurea dei pori », Studi Italiani di Filologia Classica, III S. 20, (2002), p. 104-118.

Long, A. A. \& D. N. Sedley 1987 (éd.) : The Hellenistic Philosophers. I : Translations of the principal sources with philosophical commentary, II : Greek and Latin texts with notes and bibliography, Cambridge, 1987.

MACÉ, A. 2003 : « La vitesse de la pensée : sur la pensée épicurienne de la co-affection », dans T. Bénatouïl, V. Laurand, A. Macé (éd.), L'Épicurisme antique, Les Cabiers philosophiques de Strasbourg, 15 (2003), p. 119-165.

Mansion, A. 1953 : «L'immortalité de l'âme et de l'intellect d'après Aristote », Revue philosophique de Louvain, 31 (1953), p. 444-472.

Mariotti, S. 1940 : « La "quinta essentia" nell'Aristotele perduto e nell'Accademia $\gg$, Rivista di filologia e d'istruzione classica, 68 (1940), p. 179-189.

Montarese, F. 2012 : Lucretius and his sources : a study of Lucretius, De rerum natura I 635 - 920, Berlin, 2012 (Sozomena, 12).

Moreau, J. 1939 : L'Ame du monde de Platon aux stö̈ciens, Paris, 1939.

Morel, P.-M. 1996 : Démocrite et la recherche des causes, Paris, 1996 (Philosophies antiques).

Peck, A. L. 1953: « The connate pneuma, an essential factor in Aristotle's solution of the problems of reproduction and sensation », dans E. A. Underwood (éd.), Science, Medicine and History. Essays on the Evolution of Scientific Thought and Medical Practice Written in Honour of Ch. Singer, 2 vol., London-New YorkToronto, vol. I, 1953, p. 111-121.

Pesce, D. 1974 : Saggio su Epicuro, Roma-Bari, 1974 (Biblioteca di cultura moderna, 757).

Reinhardt, K. 1953 : « Poseidonios von Apameia (3)», RE XXII, 1, 1953, col. 558-826.

SAssi, M. M. 1978 : Le teorie della percezione in Democrito, Firenze, 1978 (Biblioteca di cultura, 145).

Schrijvers, P. H. 1999 : Lucrèce et les sciences de la vie, Leiden-Boston-Köln,1999 (Mnemosyne / Supplementum, 186).

Sedley, D. N. 1976 : « Epicurus and His Professional Rivals », dans J. Bollack \& A. Laks (éd.), Études sur l'Épicurisme antique, Lille, 1976, p. 121-159 (Cahiers de Philologie, 1).

SEDleY, D. N. 1998 : Lucretius and the Transformation of Greek Wisdom, Cambridge, 1998.

Sedley, D. N. 2012 : The Philosophy of Antiochus, Cambridge, 2012.

Soleri, G. 1952 : L'immortalità dell'anima in Aristotele, Torino, 1952.

Solmsen, F. 1957 : « The Vital Heat, the Inborn Pneuma and the Aether », Journal of Hellenic Studies, 77/1 (1957), p. 119-123. DOI : 10.2307/628643

TAràn, L. 1975 (éd.) : Academica : Plato, Philip of Opus and the Pseudo-Platonic Epinomis, Philadelphia, 1975 (Memoirs of the American Philosophical Society, 107).

TAYlor, C. C. W. 1999 (trad.) : The Atomists : Leucippus and Democritus. Fragments; a text and translation with a commentary, Toronto-Buffalo-London, 1999 (Phoenix / Supplementary volume, 36 ; Phoenix / Pre-Socratics, 5).

Thivel, A. 2005: « Air, Pneuma and Breathing from Homer to Hippocrates », 
dans P. J. van der Eijk (éd.), Hippocrates in context : papers read at the XIth International Hippocrates colloquium, University of Newcastle upon Tyne 27-31 August 2002, Leiden-Boston, 2005 (Studies in Ancient Medicine, 31), p. 239-250.

Trabattoni, F. 1988 : « La dottrina dell'anima-armonia nel Fedone », Elenchos, 9/1 (1988), p. 53-74.

Verbeke, G. 1978 : « Doctrine du pneuma et entéléchisme chez Aristote », dans G. E. R. Lloyd \& G. E. L. Owen (éd.), Aristotle on Mind and the Senses. Proceedings of the VIIth Symposium Aristotelicum, Cambridge, 1978 (Cambridge Classical Studies), p. 191-214.

VERDE, F. 2013 : Elachista. La dottrina dei minimi nell'Epicureismo, Leuven, 2013 (Ancient and Medieval Philosophy, 1).

VERDE, F. 2015 : « Monismo psicologico e dottrina dell'anima in Epicuro e Lucrezio », dans E. Canone (éd.), Anima-corpo alla luce dell'etica: Antichi e Moderni, Firenze, 2015 (Lessico intellettuale europeo, 124), p. 49-64.

VERDE, F. 2016 : « Aristotle and the Garden », dans A. Falcon (éd.), Brill's Companion to the Reception of Aristotle in Antiquity, Leiden-Boston, 2016 (Brill's Companions to Classical Reception, 7), p. 35-55.

Wiersma, W. 1943 : «Die aristotelische Lehre vom Pneuma », Mnemosyne, 11 (1943), p. 102-107.

Zemplen, G. 2005 : The History of Vision, Colour \& Light Theories : Introduction, Texts, Problems, Bern, 2005 (Bern Studies in the History and Philosophy of Science, 5). 